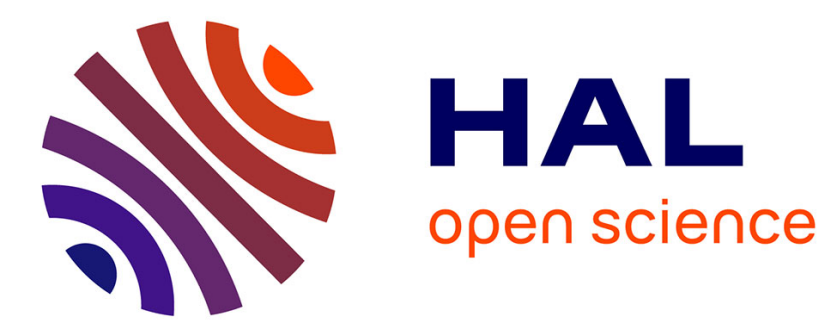

\title{
Nonlinear Analysis of Damaged Metal-Based Composite Plates Using Guided Waves
}

\author{
Yousra Baccouche, Mourad Bentahar, Charfeddine Mechri, Antonin Novak, \\ Rachid El Guerjouma
}

\section{> To cite this version:}

Yousra Baccouche, Mourad Bentahar, Charfeddine Mechri, Antonin Novak, Rachid El Guerjouma. Nonlinear Analysis of Damaged Metal-Based Composite Plates Using Guided Waves. Acta Acustica united with Acustica, 2017, 103 (6), pp.967-977. 10.3813/AAA.919126 . hal-02504356

\section{HAL Id: hal-02504356 https://hal.science/hal-02504356}

Submitted on 10 Mar 2020

HAL is a multi-disciplinary open access archive for the deposit and dissemination of scientific research documents, whether they are published or not. The documents may come from teaching and research institutions in France or abroad, or from public or private research centers.
L'archive ouverte pluridisciplinaire HAL, est destinée au dépôt et à la diffusion de documents scientifiques de niveau recherche, publiés ou non, émanant des établissements d'enseignement et de recherche français ou étrangers, des laboratoires publics ou privés. 


\title{
Nonlinear Analysis of Damaged Metallic Composite Plates Using Guided Waves
}

\author{
Yousra Baccouche, Mourad Bentahar, Charfeddine Mechri, \\ Antonin Novak, Rachid El Guerjouma
}

\author{
Laboratoire d'Acoustique de l'Université du Mans, LAUM - UMR 6613 CNRS, Le Mans \\ Université, Avenue Olivier Messiaen, 72085 LE MANS CEDEX 9, France.
}

\begin{abstract}
Resonance experiments have already proved the high level of nonlinearity in complex materials, including microcracked composites. However, the nonlinear parameters related to elastic modulus and damping are defined around a given resonance mode and are therefore known over a very limited frequency domain. In this contribution, the nonlinear parameters are determined on intact and damaged metal-based composite plates for several flexural resonances. Furthermore, the use of the theoretical formalism corresponding to guided flexural waves allowed to define a nonlinear parameter over a larger frequency domain. Finally, the nonlinear convolution method allowed to take advantage of the harmonics related to the different resonance modes in order to define new nonlinear parameters whose sensitivity is much greater than the ones determined at the fundamental frequencies.
\end{abstract}

The archived file is not the final published version of the article Y. Baccouche, M. Bentahar, C. Mechri, A. Novak \& R. E. Guerjouma (2017), "Nonlinear analysis of damaged metal-based composite plates using guided waves", Acta Acustica united with Acustica. Vol. 103(6), pp. 967-977. The definitive publisher-authenticated version is available online at

https://doi.org/10.3813/AAA.919126

Readers must contact the publisher for reprint or permission to use the material in any form. 


\section{Introduction}

Over the last two decades, studies of nonlinear dynamics in complex materials (rock, damaged materials, bone, granular media etc.) have increased markedly $[1,2,3,4$, $5,6,7,8]$. The behavior of these materials revealed to be unusual at relatively low strains. Indeed, at strain amplitudes greater than $\sim 10^{-6}$, the classical Landau theory of elasticity was found to be unable to describe the observations related to the specific scaling relations between driving strains and detected wave harmonics, resonance peak shift and the presence of conditioning $[9,10]$. Nonlinear techniques particularly probe second order effects on wave propagation at small strain levels, such as the generation of harmonics and inter-modulated frequencies, resonance frequency shift as a function of applied drive voltage, nonlinear attenuation, etc. $[11,12,13]$. In general, the observation of the higher order effects makes nonlinear parameters more sensitive to the presence of micro-cracks than the classical linear elastic parameters, which are based on velocity and attenuation changes. Among the theoretical approaches, the one based on the PM-space (PreisachMayergoyz) was able to describe the non-classical nonlinear behavior of many complex materials. The phenomenological PM-space model incorporates hysteresis into the stress-strain relationship, which makes the description of quasi-static and dynamic nonlinear behaviors possible including slow dynamics and conditioning $[14,15,16,17]$. The very limited number of physical models is related to the huge variety of mechanisms at the origin of the nonclassical elastic behavior, which could be sliding between grains in concrete [18], clapping [19], presence of water between microcracks, etc. [20, 21]. In nonlinear resonance experiments, two nonlinear hysteretic parameters $(\mathrm{NLH})$ are assessed on the basis of the changes observed in the resonance frequency and the quality factor of the monitored resonance mode with increasing strain amplitude $[9,10,12]$. However, NLH parameters are often considered in the case of a single resonance mode and hence over a very narrow frequency bandwidth. Including higher frequency components cannot be done without taking into account the dispersive nature of the excited resonances. Indeed, higher order nonlinear parameters related to higher order compression resonances have great chances to be influenced by the generated harmonics, since the frequency recovery is quite perfect. Furthermore, the application of the higher order nonlinear resonance method to characterize a localized damage will not give reliable information about the existence of a frequency dispersion related to the NLH parameters [22].
In this contribution, we present the characterization of NLH parameters through the generation of flexural waves in composite plates. Since the created damage within the composite plate is diffused, the frequency dispersion of the NLH parameters will not be influenced by the positioning of the nodes when considering higher resonance modes. Furthermore, the work presents the possibility to generalize the study to the case of the fundamental antisymmetric Lamb mode [23]. The application of a signal processing approach, namely "nonlinear convolution method" is performed in order to determine the NLH parameters related to harmonics of the excited resonances [22]. This method, which can be seen as a coupled method between "nonlinear resonance" and "harmonic generation", has proved to increase considerably the sensitivity of the proposed nonlinear method to the existence of a localized and diffused damage created within composites.

\section{Nonlinear dynamic elasticity}

In the case of a hysteretic nonlinear material, the elastic modulus can be written as

$$
\begin{aligned}
K(\varepsilon, \dot{\varepsilon})= & K_{0}(1+\beta \varepsilon(t)+\delta \varepsilon(t)+\ldots) \\
& +H(\varepsilon, \operatorname{sign}(\dot{\varepsilon}))
\end{aligned}
$$

where $K_{0}$ is the linear modulus (at weak strains), $\varepsilon$ is the strain, $\beta$ and $\delta$ represent the classical quadratic and cubic nonlinear parameters, respectively. They can be developed as a combination of $2^{\text {nd }}, 3^{\text {rd }}$ and 4 th order elastic constants. $\Delta \varepsilon$ is the local strain amplitude over the previous period, $\dot{\varepsilon}$ is the strain rate, $\operatorname{sign}(\dot{\varepsilon})=1$ when $\dot{\varepsilon}>0$ and $\operatorname{sign}(\dot{\varepsilon})=-1$ when $\dot{\varepsilon}<0$. The function $H(\varepsilon, \operatorname{sign}(\dot{\varepsilon}))$ takes into account the hysteresis in the stress-strain relationship [14, 15].

The way the nonlinear elastic modulus is expressed in Equation (1) has several interesting implications on the acoustic wave propagation. First, it can clearly differentiate between classical and hysteretic nonlinear behaviors. In a classical nonlinear system, a strain wave of frequency $f$ and amplitude $\varepsilon$ can transform into a strain wave with frequency components $2 f, 3 f$, etc. for which the amplitudes are proportional to $\varepsilon^{2}, \varepsilon^{3}$, etc. In the case of a purely hysteretic material, the second harmonic cannot be generated, and the third harmonic is found to be quadratic in the fundamental strain amplitude. 
Modelling of resonant wave experiments, based on the PM-space approach for instance, shows that when the modulus $K$ is taken into account in the wave equation, the nonlinear contribution to the solution leads to a linear decrease of the resonance frequency and the quality factor for increasing strain levels, where

$$
\begin{gathered}
\frac{f_{0}-f}{f_{0}}=C_{1} \varepsilon, \\
\frac{1}{Q}-\frac{1}{Q_{0}}=C_{2} \varepsilon,
\end{gathered}
$$

where $f$ is the resonance frequency at strain amplitude $\varepsilon$, $f_{0}$ is the linear resonance frequency at low strain amplitude $\varepsilon_{0} . Q_{0}$ and $Q$ are the quality factors measured at low $\left(\varepsilon_{0}\right)$ and higher $(\varepsilon)$ strain amplitudes. $D C_{1}$ and $C_{2}$ express the hysteretic nonlinearity existing in real $\left(\alpha_{f}\right)$ and imaginary $\left(\alpha_{Q}\right)$ components of the elastic modulus $K$ [16]. The abovementioned coefficients were determined for different materials and were found to be very sensitive to the changes existing in their microstructures $[9,17]$. In the following, we present an original approach, which allows following the frequency dispersion of $\alpha_{f}$ and $\alpha_{Q}$ by using flexural waves propagating in metal based composite plate. This was done under guided waves conditions.

\section{Theoretical models}

Flexural waves propagating in a thin plate can be described by the Bernoulli-Euler theory of beams using the following governing equation $[24,25,26]$ :

$$
E I \frac{\partial^{4} u}{\partial x^{4}}+\rho \widehat{H} \frac{\partial^{2} u}{\partial t^{2}}=0
$$

$E$ being the Young modulus, $\rho$ the density, $\widehat{H}$ the air of the cross section and $I$ is the moment of inertia of the cross section. Note that Bernoulli-Euler beam model neglects the deformation of the cross section due to the shear and rotary-inertia effects. If we consider a dynamic perturbation $u$ given by

$$
u(x, t)=U(x) \exp (\omega t),
$$

where $U$ is the amplitude of vibrations along $x$-axis and $\omega$ is the angular frequency, the dynamic beam equation can therefore be expressed as

$$
\frac{\partial^{4} U}{\partial x^{4}}-k^{4} U=0
$$

The solution of Equation (6) is written in the general form,

$$
\begin{aligned}
U(x)= & A \cosh (k x)+B \sinh (k x) \\
& +C \cos (k x)+D \sin (k x),
\end{aligned}
$$

where the wavenumber $k$ is expressed as

$$
k=\sqrt[4]{\frac{\rho \hat{H} \omega^{2}}{E I}} .
$$

The allowable values of the quantity $k$ are given by the roots $k_{i} L=m_{i}$, where $L$ is the length of the considered sample. By considering the four boundary conditions necessary to determine the relationship between the four constants of integration $A, B, C$ and $D$, the series of allowable pulses $\omega_{i}$ (and hence the resonance frequencies $\left.f_{i}=\omega_{i} / 2 \pi\right)$ are given by

$$
\omega_{i}=m_{i}^{2} \sqrt{\frac{E I}{\rho \hat{H} L^{4}}} .
$$

With the help of the Bernoulli-Euler model, we can define the velocity $V_{B}^{(i)}$ corresponding to the $i^{\text {th }}$ bending vibration, which can be expressed as a function of the thickness $h$ and the mechanical properties of the thin plate,

$$
V_{B}^{(i)}=\sqrt[4]{\frac{E \pi^{2} h^{2} f_{i}^{2}}{3 \rho}} .
$$

More recent theories have also been proposed to describe flexural waves in thin plates. Indeed, when the effect due to the shear distortion is not neglected, the Rayleigh beam equation $[27,28]$ can be written as

$$
E I \frac{\partial^{4} u}{\partial x^{4}}-\rho I \frac{\partial^{4} u}{\partial x^{2} \partial t^{2}}+\rho \widehat{H} \frac{\partial^{2} u}{\partial t^{2}}=0 .
$$

With the help of this equation, the flexural velocity can be written as

$$
V_{R}^{(i)}=\sqrt{\frac{2 E I}{\rho I+\sqrt{\rho I^{2}+\rho \hat{H} E I /\left(\pi^{2} f_{i}^{2}\right)}}} .
$$

A more complete description, proposed by Timoshenko $[29,30,31,32,33]$, can be found by taking into account the rotary-inertia and shear deformation. These parameters affect natural bending frequencies since they reduce resonance frequencies due to the growth of the inertia and flexibility of the system. Therefore, the governing equation of motion can be written as

$$
\begin{array}{r}
E I \frac{\partial^{4} u}{\partial x^{4}}-\rho I\left(1+\frac{E}{K G}\right) \frac{\partial^{4} u}{\partial x^{2} \partial t^{2}} \frac{\rho^{2} I}{K G} \frac{\partial^{4} u}{\partial t^{4}} \\
+\rho \hat{H} \frac{\partial^{2} u}{\partial t^{2}}=0 .
\end{array}
$$

$K$ and , $G$ are the shear coefficient and the shear modulus of the thin plate, respectively. Therefore, the flexural velocity using Timoshenko model is given by

$$
\begin{aligned}
V_{T}^{(i)}= & {[2 E I]^{1 / 2} \cdot[\rho I(1+E / K G)} \\
& +\sqrt{\left.\begin{array}{l}
\rho^{2} I^{2}(1+E / K G)^{2} \\
+4 E I\left(\frac{\rho \hat{H}}{4 \pi^{2} f_{i}^{2}}-\frac{\rho^{2} I}{K G\left(2 \pi f_{i}\right)^{16}}\right)
\end{array}\right]}
\end{aligned}
$$




\section{Theoretical models and nonlinear acous- tics}

Flexural velocities of the abovementioned models were compared in the case of a metal-based composite plate. Figure 1 shows that at low frequencies, the shear and rotary-inertia effects can be neglected, since the three models overlap. At higher frequencies, shear effects become more important and flexural velocities start to be different at the frequency $f_{C M} \approx 17 \mathrm{kHz}$. Therefore, it would be reasonable to consider Equation (9) as representative of the velocity dispersion corresponding to flexural waves as long as the involved frequencies are below $f_{C M}$.

The above presented theoretical models have been developed in the framework of linear elasticity, where the elastic parameters $(E, G$, etc.) do not depend on the strain generated by a standing or propagating elastic wave. Indeed, in the case of nonlinear hysteretic materials (rocks, concrete, damaged composites, etc.), Equation (1) shows that an increase of the dynamic strain will affect the materials elasticity. As a consequence, resonance frequency and Young modulus appearing in Equation (9) should be strain-dependent. Therefore, in the case of cracked materials, the flexural velocity $V^{(i)}$ should be written as

$$
V^{(i)}=\sqrt[4]{\frac{E(\varepsilon) \pi^{2} h^{2}}{3 \rho}} \sqrt{f_{i}(\varepsilon)}
$$

However, one should keep in mind that the elastic modulus is involved in Equation (14) explicitly through $E(\varepsilon)$ and implicitly through $f_{i}(\varepsilon)$. This point will be discussed later.

Finally, it is important to note that the proposed approach can also be applied in the framework of the Lamb wave theory. Indeed, Figure 2 shows the possible superimposition of the flexural wave velocities with the antisymmetric Lamb mode velocity $V A O$, which is true as long as the involved frequency remains less than $f_{C M}$.

\section{Metal-based composite sample and fa- tigue test}

\subsection{Material}

Development of metal-based composites started in the early 1970 s with a production directed primarily to the aerospace field. Since then, the range of applications of theses composites is constantly growing thanks to their excellent mechanical strength at high temperatures [34]. These composites are a combination between a low-density metal such as aluminum or titanium and reinforcements such as nano/micro particles, which offer high specific strength and stiffness on lighter materials. However, the addition of a reinforcement can weaken these alloys in terms of fatigue failure. Indeed, the presence of micro reinforcements can facilitate crack propagation within the sample and lead to significantly reduced lifetime [34].

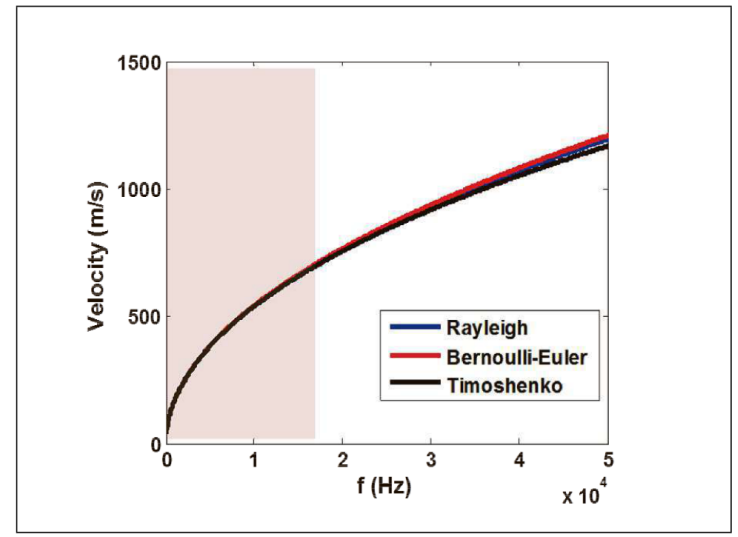

Figure 1. (Colour online) Velocity frequency dispersion by considering Timoshenko, Rayleigh and Bernoulli-Euler theories in the case of a metal-based composite TA6V ( $3 \mathrm{~mm}$ thick).

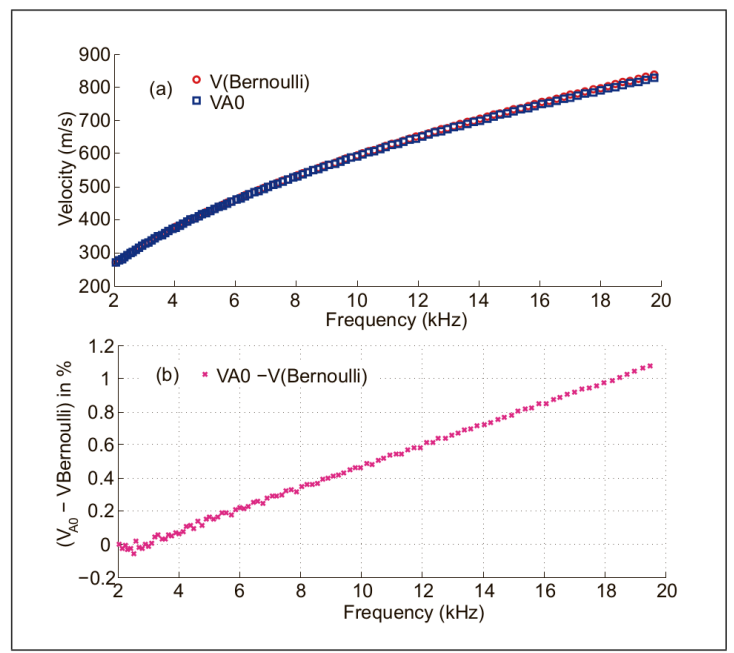

Figure 2. (Colour online) Velocities of the flexural wave and the fundamental antisymmetric Lamb wave A0 in a metal-based composite TA6V( $3 \mathrm{~mm}$ thick). The relative difference between both velocities is less than $1 \%$ below $17 \mathrm{kHz}$.

The studied composite, named TA6V, is made with a titanium matrix, which is reinforced with titanium carbide (TiC) nanoparticles. The material is elaborated by MECACHROME company in the framework of the FUI project AMETIS “Advanced Metallurgical Technologies for Innovative Systems". The project aims at undergoing technology locks that limit the emergence of nanostructuresreinforced-materials, notably through their mechanical fatigue behaviors. The studied specimens have a plate-like geometry $\left(180 \times 20 \times 3 \mathrm{~mm}^{3}\right)$. Their structure is isotropic and is characterized by the following mechanical properties: $E=(126-7 \pm 2.5) \mathrm{GPa}, v=(0.305 \pm 0.06)$, $\rho=4410 \mathrm{~kg} / \mathrm{m}^{3}$, where $E, \nu$, and $\rho$ are the Young modulus, Poisson ratio and density, respectively. 


\subsection{Fatigue test}

Composite plates are submitted to a tensile fatigue mechanical test at room temperature in order to create diffused micro-cracks. The loading steps were performed on a universal Instron ${ }^{\mathrm{TM}}$ testing machine, where the mean stress was fixed at $\sigma_{a v}=72 \mathrm{MPa}$. When $\sigma_{a v}$ is reached, the fatigue cycles are applied using a frequency and amplitude corresponding to $10 \mathrm{~Hz}$ and $\sigma_{a m p}=140 \mathrm{MPa}$, respectively. Figure 3 a shows the evolution of the number of cycles to failure as a function of the applied stress. These experimental data show that for the same $\sigma_{a v}(720 \mathrm{MPa})$ the lifetime of the sample depends on the applied $\sigma_{a m p}$ and changes from $\sim 9 \cdot 10^{3}$ to $\sim 10^{7}$ cycles.

One of the aims of the FUI project AMETIS is to test the consolidation of these metals-based composites during the fatigue tests. This has been performed through numerous fatigue tests in collaboration with MECACHROME and EADS. Indeed, fatigue tests were performed on polished samples and interrupted at different cycles in order to observe the evolution of the microstructure using a scanning electronic microscope. Figure $3 \mathrm{~b}$ represents the microstructure of the TA6V sample submitted to a fatigue test applied at $\sigma=(720 \pm 140) \mathrm{MPa}$, which is considered as the highest maximum stress (see Figure $3 a$ ). The image shows that when the fatigue test is interrupted at 6000 cycles, we observe the appearance of porosities and microcracks that did not coalesce yet to form the same macro-crack. This image, and others as well, allow to conclude that at this stage, the characterized damage is still diffused within the TA6V sample.

\section{Experimental set-up and linearity}

The experimental set up used to perform the nonlinear measurements consists of a DAQ card (16 bits dynamic resolution and up to $5 \mathrm{MHz}$ sampling frequency) connected to a power amplifier (Figure 4). DAQ card is remotely controlled to generate swept-sine signals at frequencies corresponding to the desired resonance modes at different amplitudes. Generated waveforms are amplified at $46 \mathrm{~dB}$ using a wideband power amplifier which finally excites the piezoelectric emitter transducer PZ27 attached at one extremity of the composite plate. At the other extremity, a similar transducer (PZ27) is used to receive the generated guided waves. The experimental configuration and the sample plate-like geometry favor the generation of bending resonance modes. The sample is excited around its first flexural modes at intact as well as damaged states using excitations starting from $10 \mathrm{mV}$ and amplified at $46 \mathrm{~dB}$. Note that working with harmonics of higher order modes is difficult due to the existence of mixed modes (flexural /torsion) based on boundary conditions that might affect the nonlinearity shift. Therefore, obtaining strain values for these mixed modes becomes very difficult $[6,7]$. In that sense, we have given a great attention to the boundary conditions which give repeatable frequency resonance and consequently frequency shift pa-

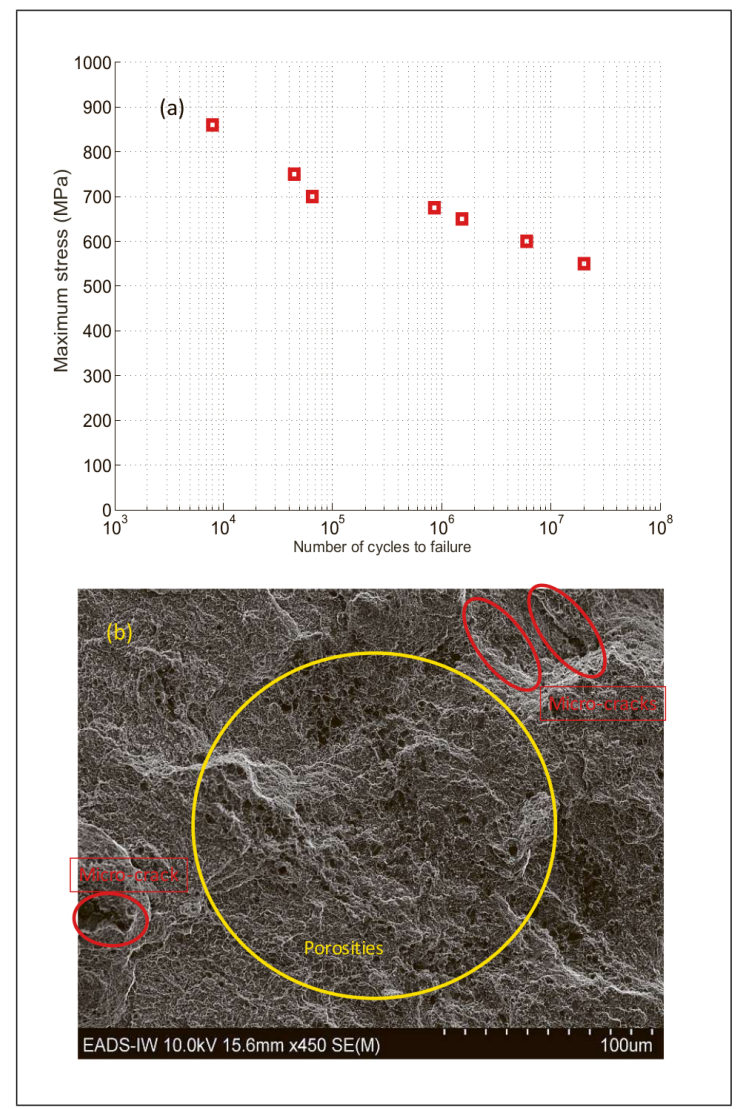

Figure 3. (Colour online) (a) Number of cycles to failure as a function of the applied mean stress on the metal-based composite TA6V; (b) The microstructure of the TA6V after 6000 fatigue cycles using a scanning electronic microscope where porosities and microcracks are present.

rameters. Among the different boundary conditions, we have verified that only two are able to give repeatable and accurate results where the frequency is measured with an error not exceeding $0.1 \mathrm{~Hz}$. The first one is when the sample is suspended and the second one is when the sample is positioned on a soft sponge put on an isolated optical table. In this contribution, we have retained the second one which is very close to the first one (the same resonance frequencies) and easy to implement in the case of samples instrumented with piezoelectric transducers. Strains were determined by measuring the displacement of each mode using a Laser Doppler Vibrometer (LDV). This procedure has been performed at every excitation frequency and level in order to take into account the changes that appear as a function of the excitation conditions. LDV displacement measurements are performed on the PZT discs. They correspond to the out-of-plane displacement component i.e. change in thickness of the PZT ceramic created by the induced strains within the composites plate. In view of the dimensions of the PZT discs $(20 \mathrm{~mm}$ diameter $\times 2 \mathrm{~mm}$ thick) the change in thickness $(\Delta e)$ of the PZT ceramic is obtained as: $\Delta e \simeq d_{33} \mathrm{~V}$, where $d_{33}$ is the piezoelectric coefficient for thickness change $\left(d_{33}=425 \mathrm{~m} / \mathrm{V}\right.$ and $V$ 
is the measured voltage across the thickness. Strains are then determined as: $\varepsilon \simeq \Delta e / e$ where $e$ is the thickness of the PZT ceramic at rest $(2 \mathrm{~mm})$. In order to obtain reliable nonlinear measurements, it is essential to verify the conditions at which the experimental setup is linear. Indeed, the detection of nonlinearity resulting from the material requires the elimination of nonlinearities related to the different components of the measurement setup such as nonlinearities due to amplification, coupling, etc. Therefore, the choice of the piezoelectric transducers, the coupling and the power amplifier becomes an important issue.

The linearity of the experimental set-up was characterized by following the evolution of amplitude and phase corresponding to monochromatic acoustic waves generated at increasing excitation levels and propagating in an intact aluminum bar. Figure 5 shows that the experimental device can be reasonably considered as linear up to an excitation voltage of $640 \mathrm{mV}$ before amplification. Indeed, within this interval both the ratio and phase difference between output and input signals at different amplitudes remain constant, where Ratio $=(-31.7 \pm 0.05) \mathrm{dB}$ and $\Delta \varphi=\left(166.2^{\circ} \pm 0.2^{\circ}\right)$. Therefore, all nonlinearities detected will be due to the wave interaction with the sample particularities as long as the excitation remains below $640 \mathrm{mV}$.

\section{Multimodal characterization of damage}

\subsection{Resonance analysis}

Depending on the excitation level, mesoscopic materials exhibit a nonlinear hysteretic behavior more or less pronounced according to the excited resonance mode. The vibration spectrum of the studied material allows determining five flexural resonance modes below $f_{C M}$. Before and after fatigue tests, we monitor the evolution of different flexural resonances in terms of resonance frequency and quality factor as a function of excitation levels. Nonlinear effects observed at damaged states allow to assess variations of resonance frequency $\alpha_{f}$ and quality factor $\alpha_{Q}$ as a function of the strain amplitude at resonance. Figure 6 shows the evolution of the NLH parameters $\alpha_{f}$ and $\alpha_{Q}$ as a function of the frequency. At the intact initial state, $\alpha_{f}$ and $\alpha_{Q}$ are equal to zero for the five resonance modes, which confirms the linearity of the experimental setup. However, at the three damaged states $(1000,5000$, and 6000 fatigue cycles), $\alpha_{f}$ and $\alpha_{Q}$ show important changes depending on the excited resonance. Indeed, at the time when the intact state didn't show any nonlinear hysteretic behavior ( $\alpha_{f} \approx 0$ and $\alpha_{Q} \approx 0$ ), we can readily notice that all the bending modes are sensitive to the induced damage at 1000 fatigue cycles. However, the sensitivity of $\alpha_{f}$ to damage is much more important than the one corresponding to $\alpha_{Q}$, where $0<\alpha_{Q}<10$ and $10<\alpha_{f}<100$. When damage cycles are increased up to 5000 fatigue cycles, we can notice that both parameters $\alpha_{f}$ and $\alpha_{Q}$ do not change significantly where their evolutions are very small for all the concerned bending modes. However, the situation is completely different at 6000 fatigue cycles. Indeed, non-

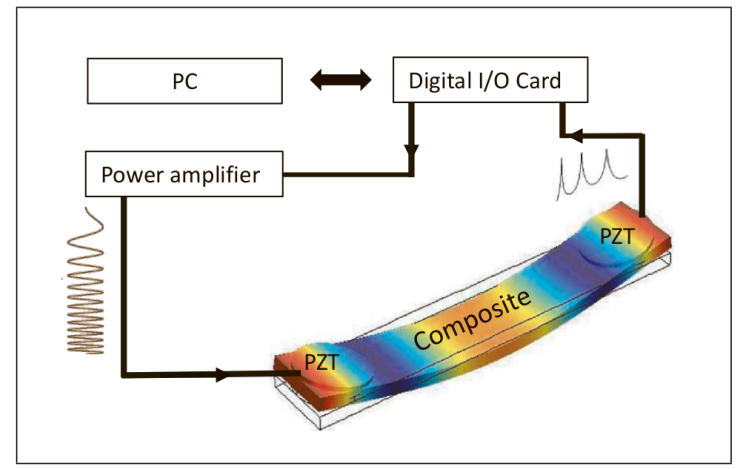

Figure 4. (Colour online) Experimental device to generate nonlinear vibrations using a dynamic resolution of 16 bits and $5 \mathrm{MHz}$ sampling frequency.

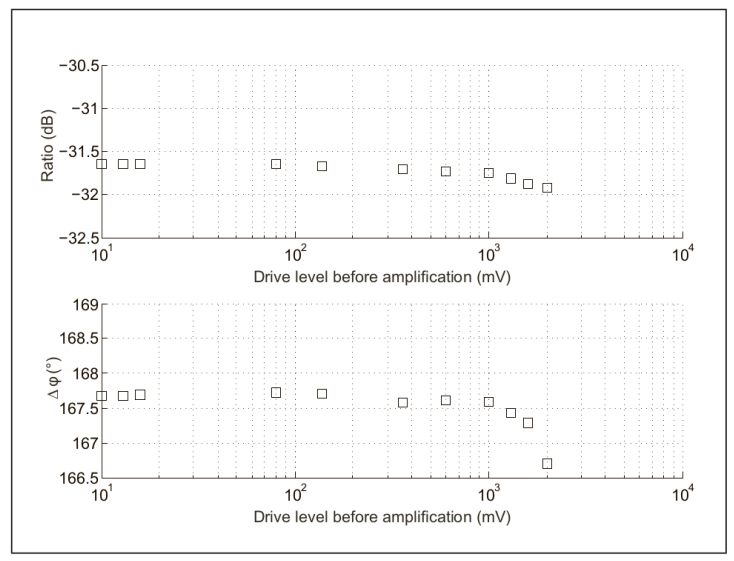

Figure 5. Linearity of the experimental device evaluated through the evolution of the ratio and phase shift between input and output signals. The system behaves linearly up to $640 \mathrm{mV}$ drive before amplification.

linear parameters reach large values at the $5^{\text {th }}$ resonance mode $\alpha_{f}=(5300 \pm 480)$ and $\alpha_{Q}=(1150 \pm 150)$. Note that nonlinear parameters $\alpha_{f}$ and $\alpha_{Q}$ related to the same bending mode do not change simultaneously. Indeed, we can see on the same figure that $\alpha_{f}$, corresponding to the 1 st bending resonance, does not change when damage is increased whereas $\alpha_{Q}$ clearly increases. When the fatigue test was conducted until the failure of the sample (1st point on Figure 3), we discovered that the high values are due to the case where an antinode of vibration is created in the vicinity of the failure zone. Note also the values extremely low of $\alpha_{f}=(28 \pm 3)$ and $\alpha_{Q}=(0.00 \pm 0.05)$ corresponding to the $2^{\text {nd }}$ bending resonance and for which a node of vibration is situated in the vicinity of the failure zone. In view of the microstructure evolution as a function of the fatigue cycles, it seems to be clear that the evolution of nonlinear parameters related to damping and elastic modulus is strongly depending on the created porosities and microcracks during the fatigue cycles as well their positions relative to nodes/ anti-nodes of the different bending 
modes. However, it is not easy to confirm, for instance, that porosities contribute to the evolution of $\alpha_{Q}$ and microcracks to the one corresponding to $\alpha_{f}$. Such a statement is out of the scope of the present contribution whose aim is to follow the evolution of damage through sensitive nonlinear acoustic parameters.

\subsection{Phase velocity analysis}

Under the conditions presented in section (4), one can use the flexural vibration spectrum to determine the dispersion of the phase velocity corresponding to the fundamental antisymmetric Lamb mode A0 as shown in Figure 7 based on Equation (14). The phase velocity presentation offers the possibility to monitor the nonlinear behavior of the TA6V composite not only for a given resonance mode through the parameters $\alpha_{f}$ and $\alpha_{Q}$ but on a larger frequency bandwidth. Indeed, we have the possibility to take advantage of the geometrical frequency dispersion observed through the five flexural resonances (Figure 6) and define a global nonlinear hysteretic parameter through the evolution of the phase velocity corresponding to the A0 mode. The A0 phase velocity is calculated based on Equation (17). However, it is important to remember that in this equation the elastic modulus intervenes explicitly through $E(\varepsilon)$ and implicitly through $f_{i}(\varepsilon)$. The experimental data allows to get easily $f_{i}(\varepsilon)$ from resonance curves at increasing amplitudes. The Young modulus is measured through the resonance frequency drop as $E=E_{0}\left(1-\Delta f\left(f_{0}\right)\right)$, where $E_{0}$ and $f_{0}$ are the resonance frequency and the Young modulus of the TA6V at rest (more details can be found in [23]).

At the weakest excitation amplitude, the slope VAO vs. $\sqrt{f}$ was found to be the same at the intact as well as damaged states. Furthermore, the slope remains the same at higher excitation levels, which means that the nonlinear signature in the phase velocity formulation is different from the one we can expect when using resonance curves. Indeed, experimental observation showed that at the damaged state the linear dependence $V A O$ vs. $\sqrt{f}$ shifts data along the same slope. Therefore, an interesting way to obtain a broadband nonlinear signature would be to define a parameter called $S$ that takes into account all the changes

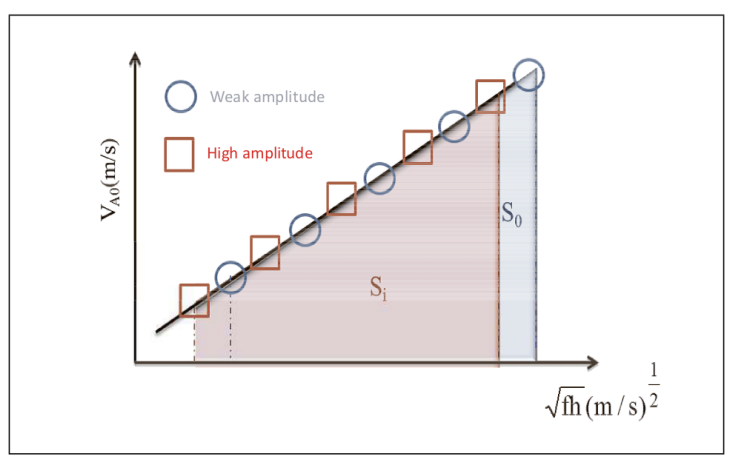

Figure 8. (Colour online) Definition of the calculated area $S$ under the velocity dispersion curve. The figure shows the evolution of the area $S$ for two excitation levels.

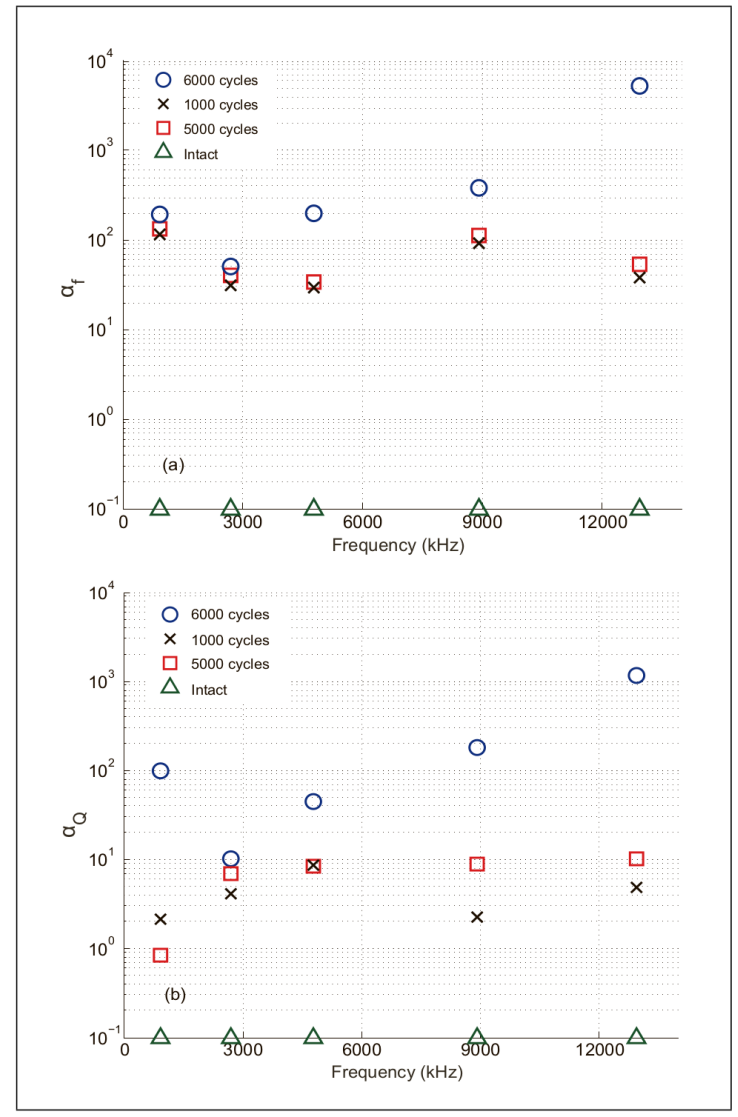

Figure 6. (Colour online) Evolution of $\alpha_{f}$ and $\alpha_{Q}$ as a function of the excited resonance mode in the case of TA6V metal-based composite sample taken at intact and damaged states at 1000 , 5000 , and 6000 fatigue cycles.

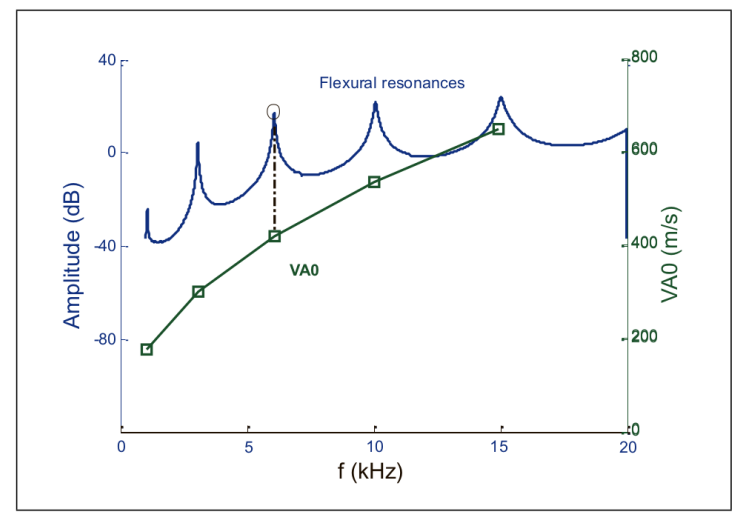

Figure 7. (Colour online) The phase velocity of the fundamental Lamb mode A0 is deduced from the flexural resonance frequencies.

observed over the five flexural resonances, where $S$ is considered at every excitation level as

$$
S(\varepsilon)=\int_{f_{1}(\varepsilon)}^{f_{5}(\varepsilon)}\left(\frac{E(\varepsilon) \pi^{2} h^{2}}{3 \rho}\right)^{1 / 4} f(\varepsilon) \mathrm{d} f,
$$


where $h$ is the thickness of the plate. Therefore, the effect of the hysteresis averaged over the excited frequency domain can be determined as $\Delta S=S\left(\varepsilon_{0}\right)-S(\varepsilon)$, where $\varepsilon_{0}$ is the weakest dynamic strain (weakest excitation amplitude). Figure 8 shows the way we have determined $S(\varepsilon)$.

Figure 9 shows the evolution of $\Delta S$ as a function of the excitation, where it can be seen that $\Delta S=0$ at the intact state. After 1000 fatigue cycles, the behavior is "linear" even at the highest excitation level. Note that when $\alpha_{f}$ is considered for each mode independently form the others, the behavior was found to be nonlinear, which means that there is a real interest in broadening the frequency domain analysis when dealing with resonances. When the 5000 fatigue cycles are reached, the behavior remains linear as long as the excitation remains below $430 \mathrm{mV}$ (before amplification). Indeed, $\Delta S$ increases with a slope $p=$ $0.016(\mathrm{~m} / \mathrm{s})^{3 / 2} \mathrm{~V}^{-1}$ (the same one found at 1000 cycles) and starts deviating at excitations greater than $\sim 430 \mathrm{mV}$ indicating thereby a change in the NLH regime over the considered frequency domain. After the 6000 fatigue cycles, the behavior is "linear" with the same slope $p$ as long as the excitation remains below $\sim 300 \mathrm{mV}$, which means that the global nonlinear behavior threshold has decreased when damage has increased. Note that the deviation from this linear behavior is done with an equivalent dynamic slope $p^{\prime}=0.033(\mathrm{~m} / \mathrm{s})^{3 / 2} \mathrm{~V}^{-1}$, which is twice greater than the slope found at 1000 fatigue cycles $\left(p^{\prime} \approx 2 p\right)$.

However, it is important to note that the variation of the NLH parameter $\Delta S$ seems to be highly dependent on the material, the distribution and the nature of the created damage. Indeed, in the case of a polymer based composite locally damaged using a three-point bending test, results were completely different. In this case $\Delta S$ is calculated for the first five bending modes using the same experimental setup mentioned in section 6 . Contrary to what we found for the metal-based composite, results presented in Figure 10 show that $\Delta S$ corresponding to the polymer composite has an asymptotic behavior which seems to be regular starting from the early excitation levels. Here we should mention that the behavior of the polymer-based composite was found to be of the same nature i.e. nonlinear from the very early excitations, when higher order bending resonances are taken into account (the first ten bending modes). Unfortunately, since we have to respect the linearity of the experimental device, we couldn't compare the first ten bending modes for both materials.

Experimental results corresponding to metal and polymer composites show that there is a real interest in broadening the frequency domain for a precocious detection of damage in heterogeneous materials. Indeed, by considering higher frequency components one can considerably decrease the geometrical effect related to the relative positioning between nodes/antinodes and cracks. Furthermore, nonlinear observations based on $\alpha_{Q}$ and $\alpha_{f}$ corresponding to a given resonance mode are qualitatively the same for both composites, but show huge differences when the considered frequency domain is larger. Finally, it is important to note that the frequency domain could also be considered

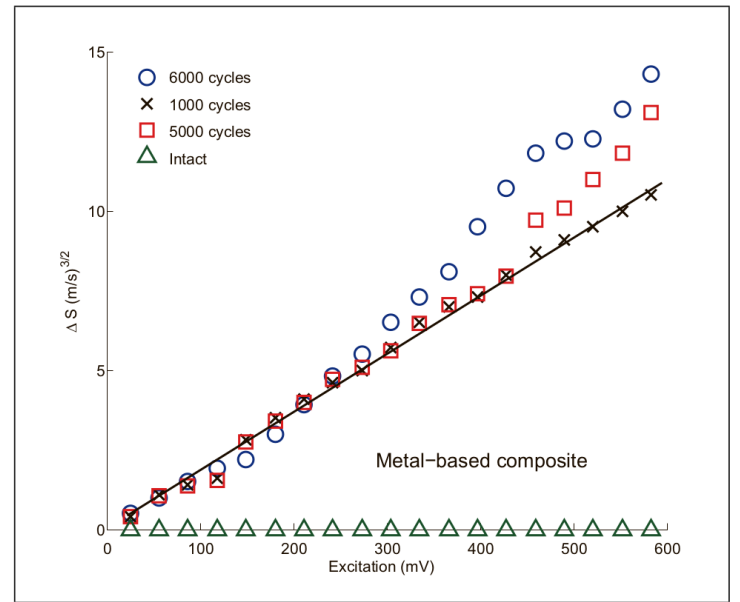

Figure 9. (Colour online) Evolution of $\Delta S$ as a function of drive level in the case of TA6V metal-based composite at intact and the three damaged states. Note the deviation from the initial linear behavior starts earlier and increases as well when fatigue cycles increase (note that $\Delta S=0$ at intact state).

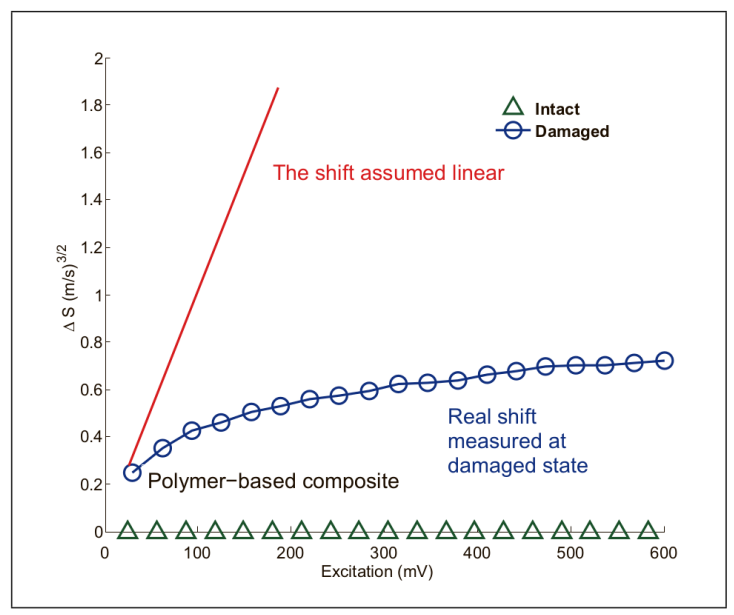

Figure 10. (Colour online) In the case of the locally damaged polymer-based composite $\Delta S$ doesn't show the linear behavior found for TA6V. In this case $\Delta S$ has a regular asymptotic evolution. Note that this result concerns the first five bending modes for a better comparison with TA6V.

differently by taking into account the higher harmonics of the excited resonances. This point will be developed in the next section.

\section{Harmonic Frequency Response of Reso- nances}

One of the most important symptoms of nonlinear systems is the appearance of higher harmonics at its output when excited by sine-like signals. The nonlinear resonance characterization presented above measures the frequency response functions by comparing input and output signals 
only at the frequency of excitation. Consequently, the information about the behavior of materials at higher harmonics is lost. It is through a generation and analysis of higher harmonics that we can determine the above mentioned NLH parameters $\alpha_{f}$ and $\alpha_{Q}$ at intermediate frequencies (between two consecutive resonances) to better assess the NLH behavior.

One way to achieve this level of analysis would be to use a spectrum analyzer to determine the harmonic frequencies related to each mono-frequency excitation. However, this type of experience is heavy to achieve for all associated frequencies to the different resonance modes that must be excited at different levels. In this section, we present a damage characterization method which couples the nonlinear resonance and harmonic generation methods providing access to original nonlinear parameters and exhibit a strong dependence on damage. The nonlinear convolution method (NLCM), idea of which was first described in $[35,36]$ with mathematical support provided later in $[22,37,38]$, is performed in order to measure simultaneously the linear acoustic frequency response function FRF of the material over one or several resonance modes and the usually out-of-reach higherorder FRF (showing the resonances of the higher harmonic modes) [22].

The method uses a so-called Synchronized Swept-Sine (SSS) signal defined as

$$
x(t)=\sin \left[2 \pi f_{1} l \exp (t / l)\right],
$$

where $l$ is the rate of frequency change defined as

$$
l=\frac{T}{\ln \left(f_{2} / f_{1}\right)} .
$$

$T$ is a duration of the swept-sine and $f_{1}$ and $f_{2}$ are initial and final frequency, respectively. The signal $x(t)$ is used as an excitation signal of the material under test.

The output signal $y(t)$ is recorded and the procedure detailed in [38] (including a Matlab ${ }^{\mathrm{TM}}$ code) is applied to extract the so-called Higher Harmonic Frequency Response Functions (HHFRF), is briefly described in Figure 11. Indeed, Figure 11a depicts the spectrogram of the input swept-sine signal (note that $y$-axis has a logarithmic scale). Figure $11 \mathrm{~b}$ depicts the spectrogram of the output signal which includes several higher harmonics created by the nonlinear system under test (a damaged state), and Figure 11c shows the HHFRF separated from each other by the NLCM. The same figure shows the fundamental vibration spectrum (blue), its second harmonic (green), and its third harmonic (red) as well. The time of the measurement equals the duration of the swept sine signal (usually few seconds). The time of processing the measured data is usually much shorter that the measurement itself. Thus, HHFRF revealed to be very fast and gives valuable information about the harmonics of the vibration modes that can be used for further analysis.

Finally, note that the existence of the harmonics spectra will not concern systematically all the vibration modes that can be generated in the fundamental vibration spectrum, since their creation depends on the interaction with the micro-cracked areas.

\section{Harmonic frequency response of reso- nances to characterize damage}

Nonlinear convolution method was applied to characterize TA6V with the help of the experimental device presented in Figure 4. By analogy to $\alpha_{f}$ and $\alpha_{Q}$ presented earlier, we can define new hysteretic nonlinear parameters corresponding to frequency and damping of the generated harmonic resonances with the help of

$$
\begin{aligned}
\alpha_{n f} & =\frac{\Delta f_{n}}{f_{n}}=\frac{1}{\varepsilon} \frac{f_{n}(\varepsilon)-f_{n 0}}{f_{n 0}}, \\
\alpha_{n Q} & =\Delta\left(\frac{1}{Q_{n}}\right)=\frac{1}{\varepsilon}\left(\frac{1}{Q_{n}(\varepsilon)}-\frac{1}{Q_{n 0}}\right) .
\end{aligned}
$$

$\alpha_{n f}$ and $\alpha_{n Q}$ are the hysteretic nonlinear parameters corresponding to the $n^{\text {th }}$ resonance harmonic. $\left(f_{n}, f_{n 0}\right)$ and $\left(Q_{n}, Q_{n 0}\right)$ are the frequencies and quality factors of the $n^{\text {th }}$ harmonic resonance at the highest and lowest excitation amplitudes, respectively.

Evolution of $\alpha_{f}$ as a function of the frequency is presented in Figure 12. These values are of the same order of magnitude than the ones found in literature, where depending on the material $\alpha_{f}$ can be $63 \pm 14$ (Pyrex) or $167 \pm 25$ (Perlite/graphite metal), see [9] for more details. Note that $\alpha_{f}$ is very dispersive since it varies between $28 \pm 5$ and

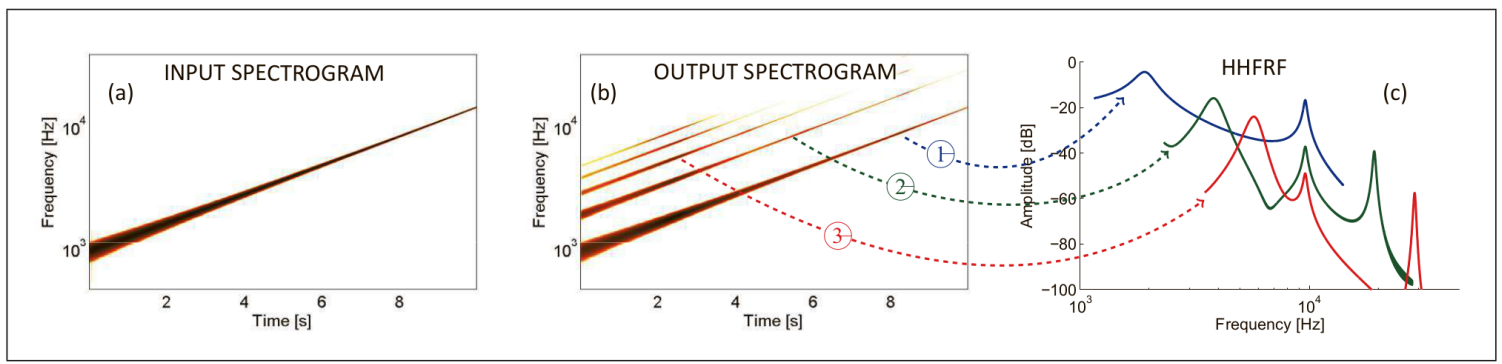

Figure 11. (Colour online) Description of the nonlinear convolution method. (a) Spectrogram of the input swept-sine signal; (b) spectrogram of the output signal including several higher harmonics; (c) Higher Harmonic Frequency Response Functions separated from each other by the NLCM method. 
$5270 \pm 100$ depending on the considered mode. This dispersion is very different from the one found in [22] in which damage was localized in space. Furthermore, the new experimental configuration allows to find resonance harmonic hysteretic parameters created by the diffused microcracks existing in the metal-based composite. Results show the existence of $2^{\text {nd }}$ and $3^{\text {rd }}$ harmonics corresponding to the third fundamental bending resonance, namely $H_{2}$ (3) and $H_{3}(3)$. We have also detected $3^{\text {rd }}$ and $2^{\text {nd }}$ harmonics corresponding to the fourth and fifth fundamental bending resonance, namely $H_{3}(4)$ and $H_{2}(5)$. The first fundamental bending modes didn't show any harmonics. Results show that $\alpha_{2 f}(3) \simeq(48 \pm 7) 10^{3}$ and $\alpha_{3 f}(3) \simeq(26 \pm 5) 10^{3}$ are much higher than $\alpha_{f}(3) \simeq(168 \pm 25)$.

The only harmonic detected for the fourth fundamental bending mode corresponds to the $3^{\text {rd }}$ harmonic, where $\alpha_{3 f}(4) \simeq(92 \pm 25) 10^{3}$, again much higher than $\alpha_{f}(4) \simeq$ $(370 \pm 25)$. Evaluation of the parameter relative to the $2^{\text {nd }}$ harmonic of the $5^{\text {th }}$ fundamental bending resonance gives $\alpha_{2 f}(5) \simeq(57 \pm 11) 10^{4}$, which is clearly greater than $\alpha_{f}(5) \simeq(5270 \pm 100)$.

Evolution of $\alpha_{Q}$ as a function of the frequency is presented in Figure 13. These values are reasonably of the same order of magnitude than the ones found in [9], where $\alpha_{Q}=(81 \pm 10)$ for the Pyrex or $\alpha_{Q}=(487 \pm 37)$ for the Perlite/graphite metal. Furthermore, the values $\alpha_{2}$ and $\alpha_{3} Q$ are clearly greater than those obtained for $\alpha_{Q}$, where $\alpha_{Q}=$ $(1150 \pm 45)$, whatever the excited mode. For the first detected harmonics, we measured $\alpha_{2 Q}(3) \simeq(17 \pm 1) 10^{3}$ and $\alpha_{3 Q}(3) \simeq(7.0 \pm 0.8) 10^{3}$. On the other hand, the parameters $\alpha_{3 Q}(4)$ and $\alpha_{2 Q}(5)$ were found as $\alpha_{3 Q}(4)=(49 \pm 3) 10^{3}$ and $\alpha_{2 Q}(5)=(157 \pm 8) 10^{3}$.

The harmonics cited above were not the only ones we have found during these experiments and they all correspond to the damage state at 6000 fatigue cycles. Indeed, other harmonics have been clearly detected and identified (ex. H2 (2), H2 (4), H3 (1), etc.) at 1000, 5000 and 6000 fatigue cycles as well but they didn't show any evolution as a function of the excitation amplitude.

Indeed, the frequency and the quality factor of these harmonics did not show any softening (frequency drop) as a function of the dynamic strain. Consequently, it turns out that resonance harmonic hysteretic parameters $\alpha_{n f}$ and $\alpha_{n Q}$ corresponding to these harmonics are zero.

However, one should note that the existence of the harmonics is in itself interesting, since it allows the calculation of other nonlinear parameters (quadratic, cubic, etc.), which are not in the scope of the present work.

\section{Conclusion}

We have discussed in this paper the determination of nonlinear hysteretic parameters $\alpha_{f}$ and $\alpha_{Q}$ by considering different resonance modes. $\alpha_{f}$ and $\alpha_{Q}$ revealed to be very dependent on the resonance mode for diffused (metal composite) and localized (polymer composite) damage [23].

In order to take into account the limitations related to the

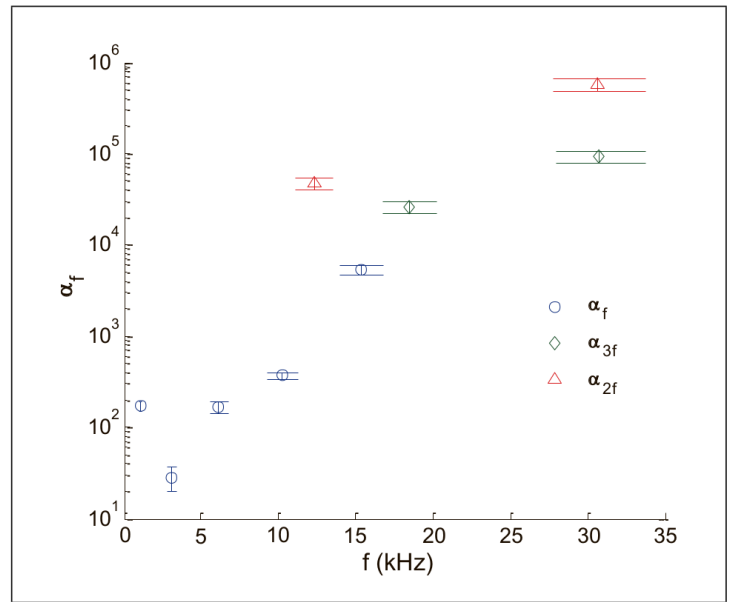

Figure 12. (Colour online) Evolution of $\alpha_{f}$ as a function of the excited resonances and their corresponding harmonics in the case of metal-based composite sample TA6V.

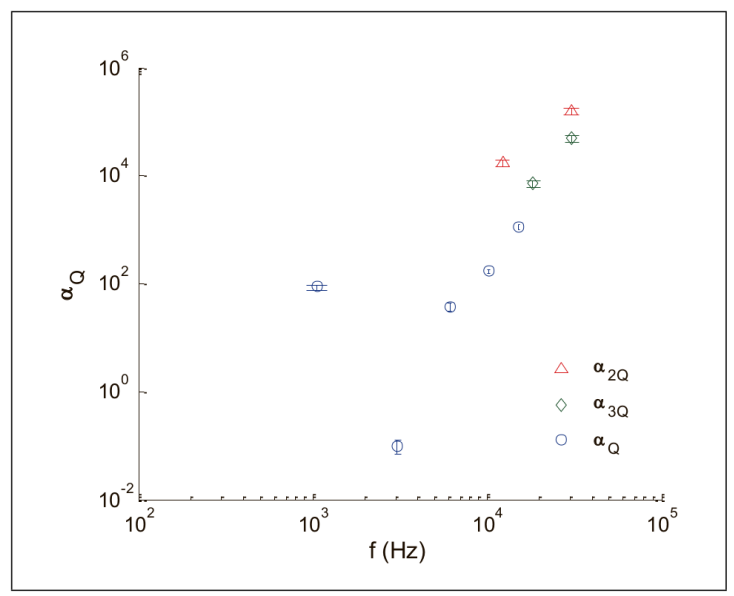

Figure 13. (Colour online) Evolution of $\alpha_{Q}$ as a function of the excited resonances and their corresponding harmonics in the case of metal-based composite sample TA6V.

relative positioning between nodes/antinodes and cracks, we proposed an evaluation of the nonlinear hysteretic behavior over a larger frequency bandwidth, which was performed using a guided wave approach. At the time where the modulus softening is qualitatively the same for all the micro-cracked materials, the approach using $\Delta S$ show that differences appear when the frequency domain is larger (Figures 9 and 10). However, the question of linking $\Delta S$ behavior to damage is still open, even though the results reported here seem to indicate that $\Delta S$ can help in understanding the global nonlinear behavior of materials in terms of nonlinear sources distribution and/or the involved micro-mechanisms. In this work, we have also determined harmonics related to the generated bending resonances and defined nonlinear hysteretic parameters $\left(\alpha_{n f}, \alpha_{n Q}, n \geq 2\right)$ 
for the harmonics whose frequencies decrease at increasing excitation level. Results show that $\alpha_{n f}$ (or $\alpha_{n Q}$ ) can be from one to several orders of magnitudes higher than $\alpha_{n f}$ (or $\alpha_{n Q}$ ). The use of harmonics in nonlinear acoustics is abundant in literature (See [6] for instance). However, these works are based on the use of compression resonances, where resonances are not dispersive and are generated at the same frequencies of the different harmonics. This makes the separation between compression modes and harmonics very difficult or may be impossible, since the amplitude of harmonics are not only due to damage present within the material but are amplified by the higher order resonances as well. The originality of this work is to use bending resonances, which are dispersive and hence offer the possibility to separate easily both contribution since the superposition between harmonics and higher order bending resonance is natural. To our knowledge, $\alpha_{n f}$ and $\alpha_{n Q}$ have only been determined for fundamental resonance modes ( $n=1)$ for compression and bending resonances, and we did not find any equivalent values corresponding to harmonics in the literature. Finally, the direct perspective of this work would be to consider nonlinear measurements based on the propagation of A0 Lamb waves propagating in plates with larger dimensions. This topic is of great interest for modern science and modern industry as well since it concerns impact or bonding defects existing within larger composite plates.

\section{Acknowledgement}

This work was supported by the project AMETIS (Advanced Metallurgical Technologies for Innovative Systems) and French Association of Women graduate from Universities (AFFDU). Authors thank Dr. Sophie Gourdet (EADS) for the high quality SEM images and Pr. M-H. Ben Ghozlen for fructuous discussions.

\section{References}

[1] J. Cantrell, W. Yost: Acoustic harmonic generation from fatigue induces dislocation dipoles. Philos. Mag. 69 (1996) 315-326.

[2] A. Moussatov, B. Castagniéde, V. Gusev: Frequency upconversion and frequency down-conversion of acoustic waves in damaged materials. Phys. Lett. A 301 (2002) 281290.

[3] V. Zaitsev, V. Nazarov, V. Tournat, V. Gusev, B. Castagniéde: Luxembourg-Gorky effect in a granular medium: probing perturbations of the material state via cross-modulation of elastic waves. Europhy. Lett. 70 (2005) 607-613b.

[4] M. Bentahar, R. E. Guerjouma: Monitoring progressive damage in polymer-based composite using nonlinear dynamics and acoustic emission. J. Acoust. Soc. Am. 125 (2009) 39-44.

[5] M. Muller, A. Sutin, R. Guyer, M. Talmant, P. Laugier, P. Johnson: Nonlinear resonant ultrasound spectroscopy (NRUS) applied to damage assessment in bone. J. Acoust. Soc. Am. 118 (2005) 3946-3952.

[6] K.-A. Van Den Abeele, J. Carmeliet, J. Tencate, P. Johnson: Nonlinear elastic wave spectroscopy (NEWS) techniques to discern material damage. Part II: Single mode nonlinear resonance acoustic spectroscopy. Res. Nondestr. Eval. 12 (2000) 31-42.

[7] Van Den Abeele, E. A. Koen, et al.: Micro-damage diagnostics using nonlinear elastic wave spectroscopy (NEWS). NDT\&E International 34 (2001) 239-248.

[8] C. Mechri, M. Scalerandi, M. Bentahar: Enhancement of harmonics generation in hysteretic elastic media induced by conditioning. Commun. Nonlinear Sci. Numer. Simulat. 45 (2017) 117-128.

[9] P. Johnson, A. Sutin: Slow dynamics and anomalous nonlinear fast dynamics in diverse solids. Acoust. Soc. Am. 117 (2005) 124-130.

[10] M. Scalerandi, S. Idjimarene, M. Bentahar, R. El Guerjouma: Evidence of microstructure evolution in solid elastic media based on a power law analysis. Commun Nonlinear Sci. Numer. Simulat. 22 (2015) 334-347.

[11] K.-A. Van Den Abeele, J. De Visscher: Damage assessment in reinforced concrete using spectral and temporal nonlinear vibration techniques. Cement and Concrete Research 30 (2000) 1453-1464.

[12] M. Bentahar, H. El Aqra, R. El Guerjouma, M. Griffa, M. Scalerandi: Hysteretic elasticity in damaged concrete: Quantitative analysis of slow and fast dynamics. Phys. Rev. B 73 (2006) 014116.

[13] P. A. Johnson, B. Zinszner, P. Rasolofosaon, F. CohenTenoudji, K.-A. Van Den Abeele: Dynamic measurements of the nonlinear elastic parameter in rock under varying conditions. J. Geophys. Res. 117 (2005) 124-130.

[14] K. R. McCall, R. A. Guyer: Equation of state and wave propagation in hysteretic nonlinear elastic materials. J. Geophys. Res. 99 (1994) 23887-23897.

[15] R. A. Guyer, P. A. Johnson: The astonishing case of mesoscopic elastic nonlinearity. Physics Today 52 (1999) 30-36.

[16] M. Scalerandi, P. P. Delsanto, P. A. Johnson: Stress induced conditioning and thermal relaxation in the simulation of quasi-static compression experiments. J. Phys. D: Appl. Phys. 36 (2003) 288-293.

[17] A. S. Gliozzi, M. Nobili, M. Scalerandi: Modelling localized nonlinear damage and analysis of its influence on resonance frequencies. J. Phys. D: Appl. Phys. 39 (2006) 38953903.

[18] T. W. Darling, J. A. TenCate, D. W. Brown, B. Clausen, S. C. Vogel: Neutron diffraction study of the contribution 
of grain contacts to nonlinear stress-strain behavior. Geoph. Res. Lett. 31 (2004) L16604.

[19] G. Shkerdin, C. Glorieux: Nonlinear modulation of Lamb modes by clapping delamination. J. Acoust. Soc. Am. 124 (2008) 3397-3409.

[20] C. Pecorari: Adhesion and nonlinear scattering by rough surfaces in con-tact: Beyond the phenomenology of the Preisach-Mayergoyz framework. J. Acoust. Soc. Am. 116 (2004) 1938.

[21] V. Gusev, N. Chigarev: Nonlinear frequency-mixing photoacoustic imaging of a crack: Theory. J. Appl. Phys. 107 (2010) 124905 .

[22] A. Novak, M. Bentahar, V. Tournat, R. El Guerjouma, L. Simon: Nonlinear acoustic characterization of microdamaged materials through higher harmonic resonance analysis. NDT\&E International 45 (2012) 1-8.

[23] Y. Baccouche, M. Bentahar, C. Mechri, R. El Guerjouma, M.-H. Ben Ghozlen: Hysteretic nonlinearity analysis in damaged composite plates using guided waves. J. Acoust. Soc. Am. 133 (2013) EL256-EL261.

[24] K. F. Graff: Wave motion in elastic solid. Dover Publications, Inc., New York, 1991.

[25] W. T. Thomson, M. D. Dahleh: Theory of vibration with applications. 5th edition. Prentice Hall, 1998.

[26] D. J. Gorman: Free vibration analysis of beams and shafts. John Wiley \& Sons, 1975.

[27] Lord Rayleigh: Theory of sound. 2nd edition. Dover Publications, New York, 1945.

[28] R. Ansari, R. Gholami, S. Sahmani: Free vibration analysis of size-dependent functionally graded microbeams based on the strain gradient Timoshenko beam theory. Composite Structures 94 (2011) 221-228.

[29] S. Timoshenko: On the correction of transverse shear deformation of the differential equations for transverse vibrations of prismatic bars. Philos. Mag. 41 (1921) 744-746.

[30] S. Timoshenko: On the transverse vibrations of bars of uniform cross-section. Philos. Mag. 43 (1922) 125-131.

[31] S. Timoshenko, D. H. Young, W. J. Weaver: Vibration problems in engineering. Wiley, New York, 1974.

[32] N. G. Stephen: The second spectrum of Timoshenko beam theory. J. Sound. Vib. 292 (2006) 372-389.

[33] R. A. Anderson: Flexural vibrations in uniform beams according to the Timoshenko theory. J. Appl. Mech. 75 (1953) 504-510.

[34] J. D. Destefani: Introduction to Titanium and Titanium alloys. ASM Handbook 2 (1990) 586-591.

[35] A. Farina: Simultaneous measurement of impulse response and distortion with a swept-sine technique. Proc. AES 108th Conv., Paris, France, 2000.

[36] A. Farina, A. Bellini, E. Armelloni: Non-linear convolution: A new approach for the auralization of distorting systems. Proc. AES 110th Conv., Amsterdam, The Netherlands, 2001.

[37] A. Novak, L. Simon, F. Kaldec, P. Lotton: Nonlinear system identification using exponential swept-sine signal. IEEE Transactions on Instrumentation and Measurement 59 (2010) 2220-2229.

[38] A. Novak, P. Lotton, L. Simon: Synchronized swept-sine: Theory, application and implementation. J. Audio Engineering Society 63 (2015) 786-798. 